\title{
Dynamic Data Streaming for an Appliance
}

\author{
Marta Patiño and Ainhoa Azqueta \\ Laboratorio de Sistemas Distribuidos, E.T.S. Ingenieros Informáticos, Universidad Politécnica de Madrid, Spain
}

Keywords: Data Stream Processing, NUMA Aware, Appliances.

\begin{abstract}
Many applications require to analyse large amounts of continuous flows of data produced by different data sources before the data is stored. Data streaming engines emerged as a solution for processing data on the fly. At the same time, computer architectures have evolved to systems with several interconnected CPUs and Non Uniform Memory Access (NUMA), where the cost of accessing memory from a core depends on how CPUs are interconnected. In order to get better resource utilization and adaptiveness to the load dynamic migration of queries must be available in data streaming engines. Moreover, data streaming applications require high availability so that failures do not cause service interruption and losing data. This paper presents the dynamic migration and fault-tolerance capabilities of UPM-CEP, a data streaming engine designed to take advantage of NUMA architectures. The preliminary evaluation using Intel HiBench benchmark shows the effect of the query migration and fault-tolerance on the system performance.
\end{abstract}

\section{INTRODUCTION}

Large companies use mainframes for running their application businesses. In general, the main frames are characterized by having a large memory and several cores. The mainframe usually runs the operational database which is part of the core business of companies.

The goal of the CloudDBAppliance project ${ }^{1}$ is setting up a European Cloud Appliance that integrates three data management technologies: operational database, analytical engine and real-time data streaming on top of a many-core architecture with hundreds of cores and several Terabytes of RAM provided by Bull.

Servers with several CPUs in a single board, many cores, with non-uniform memory access (NUMA) and $128 \mathrm{~GB}$ or more are common these days.

Nowadays several data streaming engines (DSE) are available and ready to be used such as Flink (Fundation, T. A. (2014)), Spark Streaming ${ }^{2}$, and Storm (Foundation, A. S. (2015)) among others. These data streaming engines were designed to run on a distributed system made of several computers connected through a network in a LAN in order to scale and process large amount of events per second. However, mainframes although their architecture

\footnotetext{
${ }^{1}$ The CloudDBAppliance Project. http://clouddb.eu
}

resemble a distributed architecture they expose a centralized architecture with no network communication and large shared memory. In this paper we present UPM-CEP, a NUMA aware DSE for appliances. UPM-CEP provides a scalable architecture to be deployed on NUMA architectures. To the best of our knowledge, this is the first DSE with this feature. The paper describes the UPM-CEP migration and fault-tolerance features and preliminary performance results using the Intel HiBench benchmark.

The rest of the paper is organized as follows. Section 2 introduces NUMA architectures. Section 3 presents data streaming engines main features. The architecture of UPM-CEP is presented in Section 4. Section 5 presents the dynamic migration algorithm, while Section 6 presents the fault-tolerance protocol. The performance evaluation is shown in Section 7 and finally, Section 8 presents the conclusions and future work.

\section{NUMA ARCHITECTURES}

A NUMA system consists of several connected CPUs, also called nodes or sockets. Each CPU has its own memory that can be accessed faster than the

\footnotetext{
${ }^{2}$ https://spark.apache.org/streaming/
} 
memory attached to other CPUs. Main vendors have implemented this model and connect the CPUs using QuickPathInterconnect (QPI, Intel) or other means, like HyperTransport in AMD processors. The cores of a CPU have its own L1 and L2 caches and share a L3 cache. In the case of Intel each CPU has a number of QPI links used to connect to other CPUs. Depending on the total number of CPUs the memory of other CPUs is reachable in a single hop or more hops are needed.

For instance, the Bullion S16 consists of 16 Intel NUMA nodes each of them equipped with 18 cores. Figure 1 shows how the CPUs are connected and Figure 2 shows the memory distance between different NUMA nodes.

The distance represents the relative latency for accessing the memory from one CPU to another one. For instance, a program in node 0 accessing data in its own memory has a cost of 10, while accessing the data in the memory attached to node 1 has a higher cost, 15 (50\% overhead). If data is the memory of any of the 14 remaining nodes, the cost is 40 . That is, it costs 4 times more than accessing local memory of node 0 .

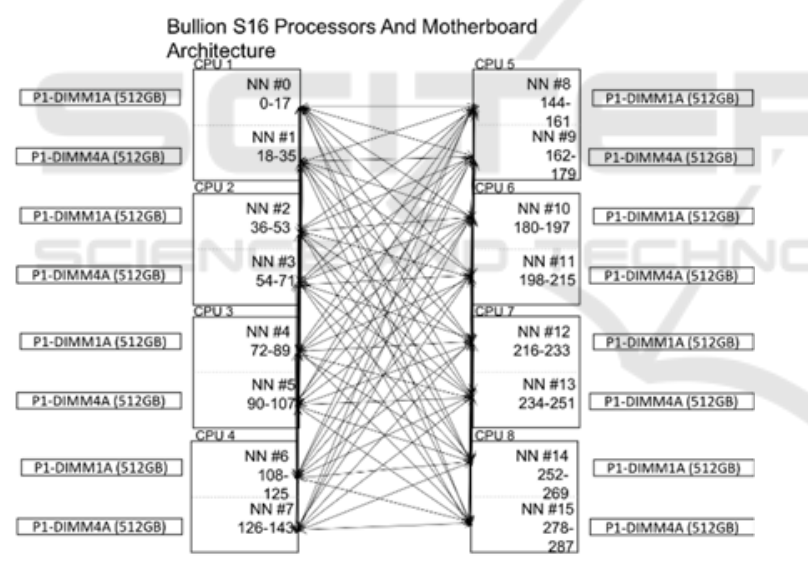

Figure 1: Bullion S16 Architecture.

Bullion S16 Latency Access to Different NUMA nodes

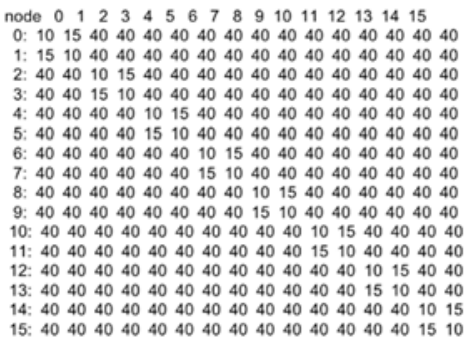

Figure 2: NUMA distances in the Bullion S16.
Operating systems in general allocate threads on the CPU with lowest usage. Therefore, the threads of a process can be spread across several CPUs and therefore the performance of the application can be affected by remote access to the data. Linux systems provide functions to bound threads to a CPU (e.g., libnuma) and even tools to bind a process to a CPU (e.g., numactl). The numactl command also allows to define where the memory is allocated for an application, for instance, on a single CPU, on a set of CPUs or interleaved among a set of CPUs.

\section{DATA STREAMING ENGINES}

Stream Processing (SP) is a novel paradigm for analysing data in real-time captured from heterogeneous data sources. Instead of storing the data and then process it, the data is processed on the fly, as soon as it is received, or at most a window of data is stored in memory. SP queries are continuous queries run on a (infinite) stream of events. Continuous queries are modeled as graphs where nodes are SP operators and arrows are streams of events. SP operators are computational boxes that process events received over the incoming stream and produce output events on the outgoing streams. SP operators can be either stateless (such as projection, filter) or stateful, depending on whether they operate on the current event (tuple) or on a set of events (time window or number of events window). Several implementations went out to the consumer market from both academy and industry (such as Borealis (Ahmad, 2005), Infosphere (Pu, 2001), Storm (Foundation, 2015), Flink (Fundation, Apache Flink ${ }^{\circledR}$ - Stateful Computations over Data Streams, 2014) and StreamCloud (Gulisano, StreamCloud: An Elastic and Scalable Data Streaming System, 2012)). Storm and Flink followed a similar approach to the one of StreamCloud in which a continuous query runs in a distributed and parallel way over several machines, which in turn increases the system throughput in terms of number of tuples processed per second. UPM-CEP adds efficiency to this paralleldistributed processing being able to reach higher throughput using less resources. It reduces the inefficiency of the garbage collection by implementing techniques such as object reutilization and takes advantage of the novel Non Uniform Memory Access (NUMA) multicore architectures by minimizing the time spent in context switching of SP threads/processes. 


\section{UPM-CEP DATA STREAMING ENGINE}

UPM-CEP provides a client driver for streaming applications, the JCEPC driver that hides from the applications the complexity of the underlying system. Applications can create and deploy continuous queries using the JCEPC driver as well as register to the source streams and subscribe to output streams of these queries. During the deployment of a streaming query the JCEPC driver takes care of splitting the query into sub-queries and deploys them in the CEP. Some of those sub-queries can be parallelized. For instance, the query in Figure 3 shows a data streaming query made out 2 input streams and 8 operators. The operators are either stateless (SL) or stateful (SF) operators.

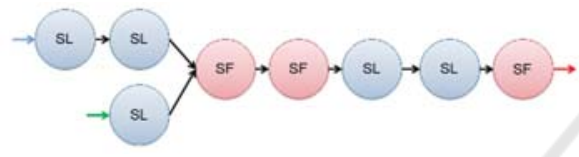

Figure 3: Data streaming query.

UPM-CEP has several stateless and stateful operator implemented and users can create their own customized operators. The stateless operators that can be found are: 1) Map: that allows to select the desired fields from the input tuple and create an output tuple with those fields. 2) Filter: Only the tuples that satisfy a defined condition are sent through the output stream, the rest of tuples are discarded. 3) Demux: sends the input tuples to all the output streams that satisfies the defined conditions and 4) Union: Tuples that arrives to the operator from different input streams are sent to one output stream.

Regarding the stateful operators two window oriented operators are available: 1) Aggregate: group all tuples that are in the time or size window taking into account defined functions executed over the fields of all the tuples. Moreover, tuples can be grouped into different windows if the group by parameter is specified. 2) Join: Correlates tuples from two different input stream. Two time windows are created, one per input stream and when the windows are slide tuples are joined creating one output tuple taking into account a specified predicate.

UPM-CEP partitions queries into subqueries so that, each subquery executes in a different node. Figure 4 shows how the previous query is splitted into four subqueries (SQ1, SQ2, SQ3 and SQ4). The number of subqueries of a given query is defined by the number of stateful operators. All consecutive stateless operators are grouped together in a subquery till a stateful operator is reached. That stateful operator is the first operator of the next subquery. This way of partitioning queries has proven to be efficient in distributed scenarios (Gulisano, StreamCloud: A Large Scale Data Streaming System, 2010). We have applied the same design principles to UPM-CEP although it is not a distributed setup, the same principles apply minimizing the communication across NUMA nodes in this case and keeping the same semantics a centralized system will provide.

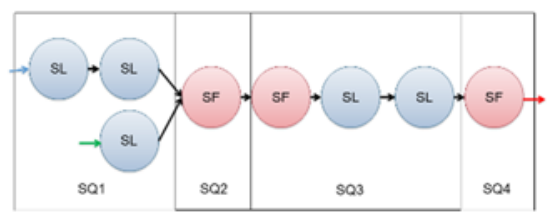

Figure 4: Query partitioning.

Subqueries can be parallelized in order to increase the throughput. Each instance of a subquery can run in a different core in the same node. Figure 5 shows how subqueries of the previous example could be parallelized. There are 3 instances of SQ1, one instance of SQ2, two instances of SQ3 and three instances of SQ4.

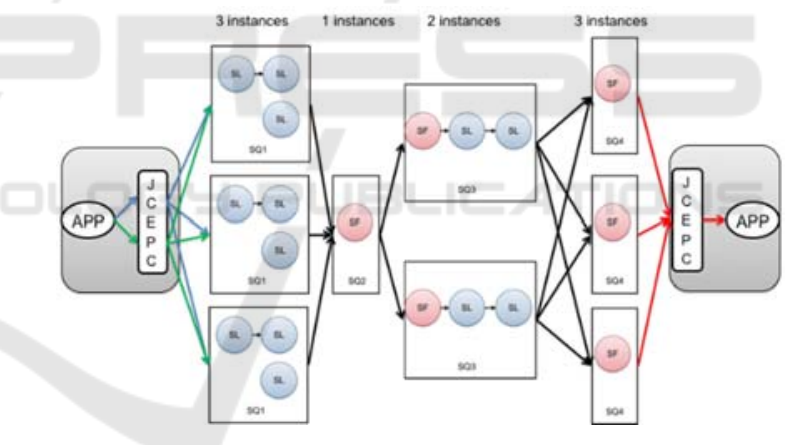

Figure 5: Query parallelization.

The main challenge in query-parallelization is to guarantee that the output of a parallel execution is the same as a centralized one. If we consider a sub-query made by only one operator, this challenge means that the output of a parallel operator must be the same as a centralized operator. On the other hand, window oriented operators require that all tuples that have to be aggregated/correlated together are processed by the same CEP instance. For example, if an Aggregate operator computing the total monthly operations of the bank accounts for each client is parallelized over three CEP Instances, it must be ensured that all tuples belonging to the same user account must be processed by the same CEP Instance in order to produce the correct result. 
To guarantee the equivalence between centralized and parallel queries, particular attention must be given to the communications among sub-queries. Consider the scenario depicted in Figure 6 where there are two sub-queries, Sub-query 1 and Sub-query 2, with a parallelization degree of two and three, respectively. If Sub-query 2 does not contain any window oriented operator, CEP instances at Subquery 1 can arbitrary decide to which CEP instance of Sub-query 2 send their output tuples. Output tuples of Sub-query 1 are assigned to buckets. This assignment is based on the fields of the tuple. Given $B$ distinct buckets, the bucket $b$ corresponding to a tuple $t$ is computed by hashing one or more fields of the tuple modulus $B$. All tuples belonging to a given bucket are sent to the same CEP instance of the Sub-query 2.

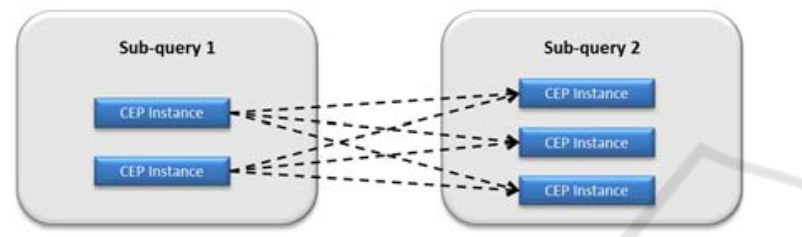

Figure 6: Subquery Connections.

The fields to be used in the hash function depend on the semantics of the window oriented operators defined at Sub-query 2 and the mapping among buckets and downstream CEP Instances depends on the load balancing algorithm used in the CEP.

- Join: if the join predicate has at least one equality condition, it is an equi-join (EJ), otherwise it is a cartesian product. For downstream operators of type EJ, the hash function is computed over all the fields used in the equalities plus the optional fields which could appear in the group-by clause.

- Aggregate: the fields used in the hash function are all the fields used in the group-by parameter. In this way, it is ensured that all tuples sharing the same values of the attributes specified in the group-by parameter are processed by the same CEP instance.

UPM-CEP comes with this default partition strategy used for splitting a query into sub-queries in the absence of user defined split policies. According to this strategy a new sub-query is created anytime one of the following conditions is satisfied during the query:

- It is a stateful operator.

- It is an operator with more than one input stream.

All the event oriented operators before the first stateful operator are part of the same sub-query.

\subsection{UPM-CEP Architecture}

The UPM-CEP architecture consists of two main components: the orchestrator and instance managers. Other components are the reliable registry (Zookeeper) and the metric server. Figure 7 depicts how the CEP components can be deployed in a scenario with several NUMA nodes or nodes.

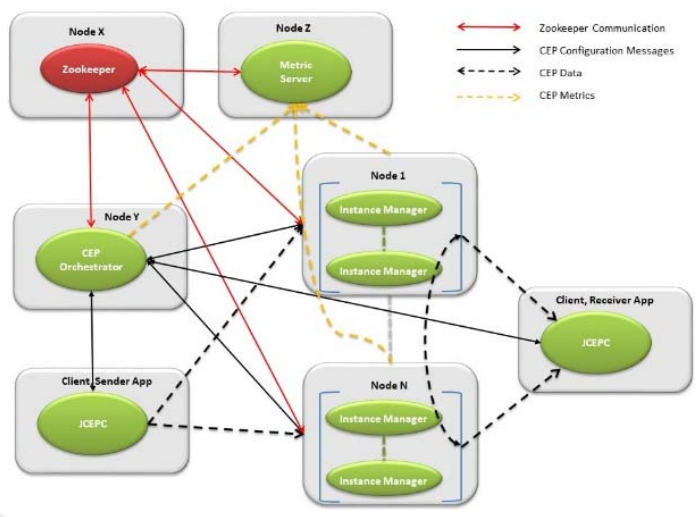

Figure 7: CEP Components.

\subsubsection{Orchestrator}

The orchestrator is in charge of managing the rest of the elements of the CEP. There is only one instance of this component in a deployment. It deploys queries and subqueries in the instance managers and balances the load among different nodes running instance managers.

The state of the orchestrator is kept in Zookeeper (Fundation, Apache ZooKeeper, 2010) so that, if there is a failure a new orchestrator can be run and take its state from Zookeeper. Active replication could be an alternative design however, although fault-tolerance is easier to implement in this case, the overhead of active replication of the orchestrator will have an impact on regular processing (when there are no failures).

\subsubsection{Instance Managers}

The instance manager is the component in charge of running queries. Each instance manager runs on a core of NUMA node and can run one or more subqueries. Instance managers are single threaded.

Instance managers receive tuples either from clients (through the JCEPC driver) or from other instance managers. Instance managers must be aware of the nature of the subquery it sends tuples. In a scenario in which there is no parallelism, an instance manger running a subquery will send all the data to the next subquery. These means, tuples from the first 
subquery are sent directly to the next one, this type of tuple sender process is called point to point balancer. Figure 8 shows two subqueries $\mathrm{SQ}_{1}$ and $\mathrm{SQ}_{2}$, both are deployed using one instance manager. And tuples t1 to t6 are being sent from $\mathrm{SQ}_{1}$ to $\mathrm{SQ}_{2}$ by means of the point to point balancer.

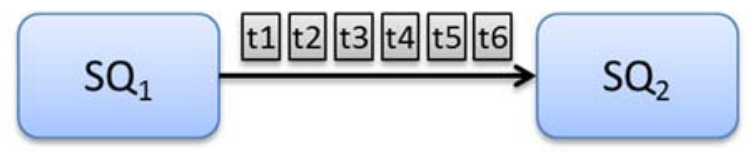

Figure 8: Point to Point Balancer.

However, if $\mathrm{SQ}_{2}$ is deployed in several instances, $\mathrm{SQ}_{1}$ has to take into consideration the type of operator is placed at the beginning of $\mathrm{SQ}_{2}$. If it is a stateless operator, tuples from the previous instance manager are sent in a round-robin fashion. For instance, Figure 9 represents the aforementioned scenario. $\mathrm{SQ}_{2}$ has been parallelized in three different instances and the first operator is stateless, these means the tuples can be handle by any of the $\mathrm{SQ}_{2}$ instances. The round robin balancer sends tuples t 1 to t6 as presented, tuple $\mathrm{t} 1$ is sent to the first instance, $\mathrm{t} 2$ is sent to the second instance, $t 3$ is sent to instance number 3 and the process is repeated with tuples $t 4$ to t6 beginning from the first instance.

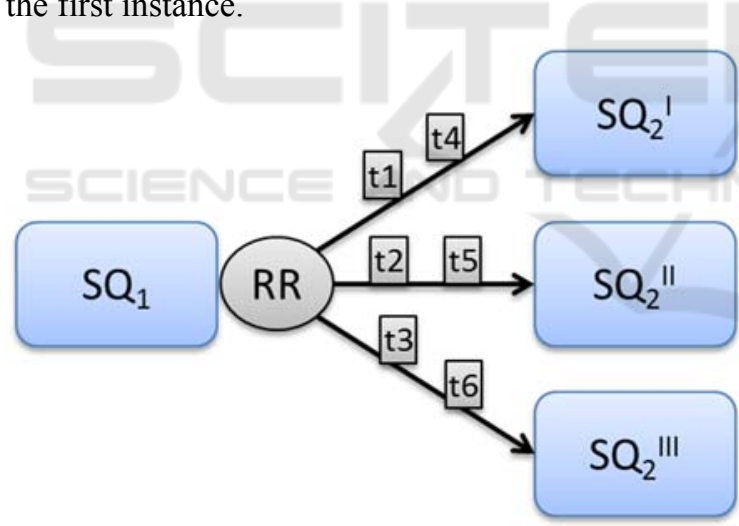

Figure 9: Round Robin Balancer.

Nevertheless, if the first operator is stateful these means that tuples have to be handled by a specified instance. For that cases a route key balancer is required, taking into account the group by clause specified in the operator configuration. Tuples produced by $\mathrm{SQ}_{1}$ are routed to the required $\mathrm{SQ}_{2}$ instance, Figure 10 shows how the route key balancer works sending tuples t1 to t6 to the different instances of $\mathrm{SQ}_{2}$. Tuple $\mathrm{t} 6$ is sent to $\mathrm{SQ}_{2}{ }^{\mathrm{I}}$, tuples $\mathrm{t} 1, \mathrm{t} 2$ and $\mathrm{t} 4$ are sent to $\mathrm{SQ}_{2}{ }^{\mathrm{II}}$ taking into account the route key and finally tuples $\mathrm{t} 3$ and $\mathrm{t} 5$ are sent to $\mathrm{SQ}_{2}{ }^{\mathrm{III}}$.

To complete the types of balancer presented, the UPM-CEP also defines a broadcast balancer of those operators that requires to send the tuples to all the instances. Figure 11 exposes an example of how tuples are sent from $\mathrm{SQ}_{1}$ to all $\mathrm{SQ}_{2}$ instances.

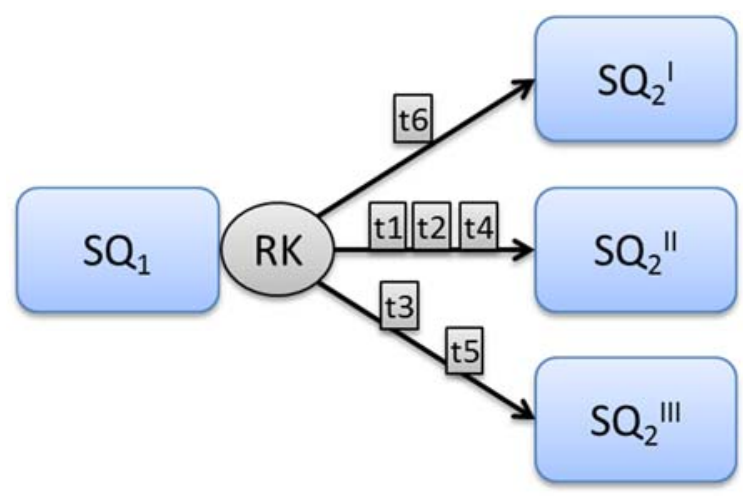

Figure 10: Route key Balancer.

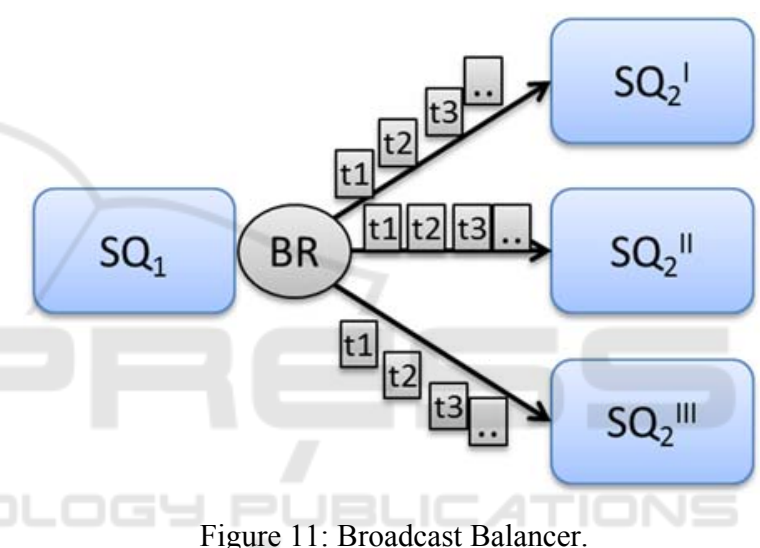

Figure 11: Broadcast Balancer.

\section{DYNAMIC MIGRATION}

The metric server component of the DSE stores all the metrics related to the performance and resource usage of the DSE. That is, input load, throughput and latency of each operator, subquery and query running in the system. Regarding the resource usage the DSE monitors CPU, memory and bandwidth consumed by each Instance Manager. If an Instance Manager is saturated, dynamic migration will try to move one of the subqueries running on that IM to another less loaded IM.

Dynamic migration also happens when a new Instance Managers are provisioned to the DSE, or when moving sub-queries from an Instance Manager running on one NUMA node to another one. In the first case, the goal of moving a sub-query is to distribute the load between an overloaded Instance Manager and a new one. In the second case, the reconfiguration can move two sub-queries that are 
exchanging data with high frequency on the same NUMA node in order to take advantage of the local memory.

We defined a migration protocol for the DSE: State Transfer. To explain the migration protocol, we will use the query depicted in Figure 12. In the figure there is a query $\mathrm{Q}$ with three sub-queries named SQ A, SQ B and SQ C. SQ A and SQ C are deployed with 2 instances each and SQ_B is deployed using 3 instances. Each sub-query is deployed in its own Instance Manager. $\mathrm{C}$ and $\mathrm{R}$ represent respectively the client application sending tuples and the receiver application waiting for the query results. Moreover let us assume that we want migrate SQ_B 2 from IM4 to IM8.

The completion time for the State Transfer protocol depends on the state size, that is, the number of tuples kept in the window structures at the time the reconfiguration is issued.

\section{State Transfer Protocol:}

1. The protocol starts deploying a new Instance of SQ_B (SQ_B $\left.B_{2 N E W}\right)$ in the Instance Manager IM8.

2. Then, the upstreaming sub-queries, $S Q A_{1}$ and SQ_ $A_{2}$ are informed that the sub-query $S Q B_{2}$ will be moved from IM4 to IM8. As a result of this action, the IMs running SQ_ $A_{1}$ and $S Q A_{2}$ flush their output streamss, and turn in a reconfiguration mode where they start buffering all new output tuples with destination SQ_B $\mathrm{B}_{2}$.

3. IM4 takes a snapshot of the state (if any) of SQ_B $B_{2}$ and transfers it to the new instance SQ $B_{2 N E W}$ running in IM8.

4. Once the state is transferred, $S Q A_{1}$ and $S Q \_A_{2}$ first send to SQ $B_{2 N E W}$ all the tuples stored in the meanwhile in the buffers and then turn themselves back in the normal operation mode.

\section{FAULT-TOLERANCE}

The simplest solution to provide high availability in a datacentre is simply to deploy the DSE in another appliance (backup) and resume the processing of tuples in that appliance if the active one fails (primary). A replication manager is needed for detecting the failure of the primary appliance. The replication manager also sends the queries to be deployed to the backup. These queries are registered in the backup during regular processing. When the primary fails in a primary-backup scenario in a data centre, the backup will redeploy all the queries and resume the processing.
This solution is valid if some tuples can be lost and this does not affect the application. However, if no tuple should be missed then, either an active replication approach is followed or some check pointing mechanisms must be in place. In that case some mechanisms are needed to store the tuples so that they can be replayed in case of a failure. If tuples are replayed, two semantics are possible: at least once or exactly once. That is, tuples are processed exactly once (there are no duplicates in case of failures) or there can be duplicates (at least once). The exactly once policy is more expensive as it needs to register every outcome. The DSE currently implements at least once semantics by implementing active replication. That is, all events are sent to the two appliances, and both of them process all events. The sinks that receive the outcome of the data streaming engine will receive output events from the two appliances and filters them in order to avoid sending duplicates to the client during regular processing. When a failure happens, one of the appliances will stop sending output events. At that point the sink will receive events from one appliance and send these results to the client. In this scenario there will be no duplicate outcomes (exactly once guarantee).

\section{PERFORMANCE EVALUATION}

UPM-CEP performance has been measured using the Intel HiBench benchmark (Intel, 2017). This benchmark allows to evaluate different big data frameworks and contains 19 different workloads that are distributed in: micro, machine learning, sql, graph, websearch and streaming. Specifically, we focus on the streaming workloads: 1) Identity: This workload reads input tuples and produces the same tuples without any modification. A map operator is defined with the same input and output fields. 2) Repartition: Modifies the parallelism level and distributes the load in a round robin fashion. It defines a map operation that copies the input to the output.

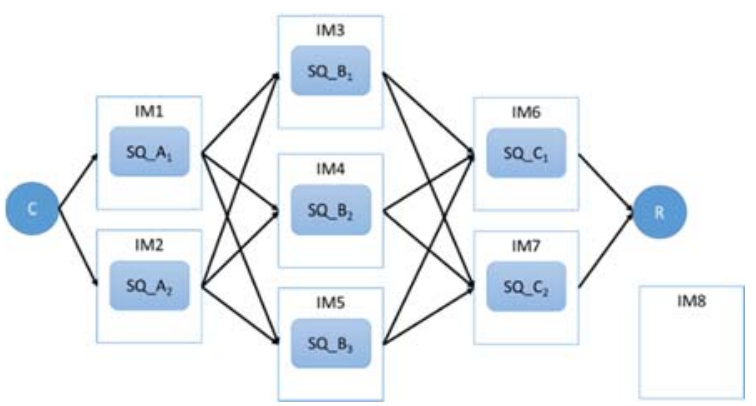

Figure 12: Example query for the migration protocol. 
The query is deployed several times. Tuples are sent to the different instances of the query in a round robin fashion. 3) Stateful wordcount: counts the number word. This workload requires several operators, first of all a map operator picks only the word from the input tuple; an aggregate operator with a number of tuples window and a group by condition based on the word is added. This query tests the route key balancer. 4) Fixed Window: This workload tests the performance of the time window operator group by a field.

This streaming workload has been implemented to be executed in four different streaming frameworks such as Flink, Storm, Spark and Gearpump. We have implemented same workloads for the UPM-CEP. In this evaluation we use the Fixed Window query, which aggregates the connections to a server from each IP address during a period of time. After this time expires, a tuple with the timestamp of the first and last connection from that IP address and the number of connections during that period is produced.

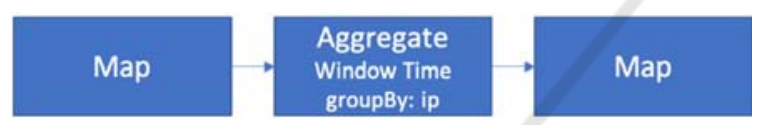

Figure 13: HiBench Fixed Window Topology.

This query, represented in Figure 13, is implemented as a map operator that selects the IP address and the connection time (timestamp) from incoming tuples. Then, an aggregate operator with a time window of 30 seconds per IP is defined. When the window is triggered a tuple is emitted with the timestamp of the first tuple in the window and the number of tuples. To finalize a map operator, add an extra field to the tuple with the timestamp at this moment. The code below corresponds to the aggregate function.

AggregateOperatorConfig aggregator $=$ new

AggregateOperatorConfig("aggregator",

\section{PROJECTOR_STREAM, AGGR_STREAM);}

aggregator.setWindow(OperatorEnums.WindowTyp

e.TIME, wsize, wadv);

aggregator.addGroupByField("ip");

aggregator.addIntegerFunctionMapping("counter",

OperatorEnums.Function.COUNT, "ip");

aggregator.addLongFunctionMapping("startts",

OperatorEnums.Function.LAST_VAL,

ParameterStore.TIMESTAMP_USER_FIELDNAM
E);

aggregator.addStringFunctionMapping("ip",

OperatorEnums.Function.LAST_VAL, "ip");

query.addOperator(aggregator);

The goal of the evaluation is to show the performance during load balancing and when failures occur.

For the replication protocol we show the throughput and latency of subquery 2 , the one starting with the aggregate operator before the failure, while the migration happens and after the system is reconfigured. Figure 14 shows those values before the migration happens. At that point 10,000 tuples are received by this subquery. The response time is 0.04 ms.

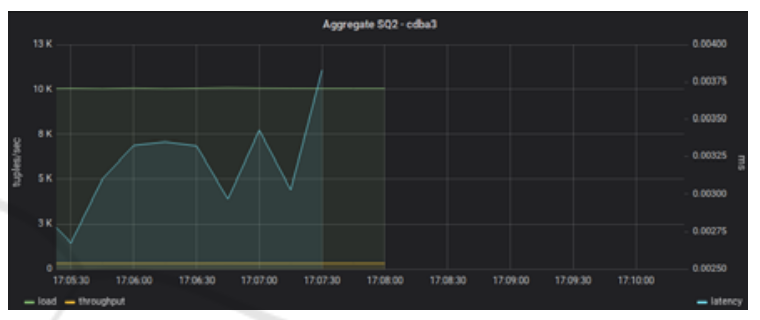

Figure 14: Subquery migration, Load and response time before the migration.

Then, the query is migrated to another node. Figure 15 shows the load and response time after the query is migrated. It takes around 20 seconds to transfer the state and start processing tuples again.

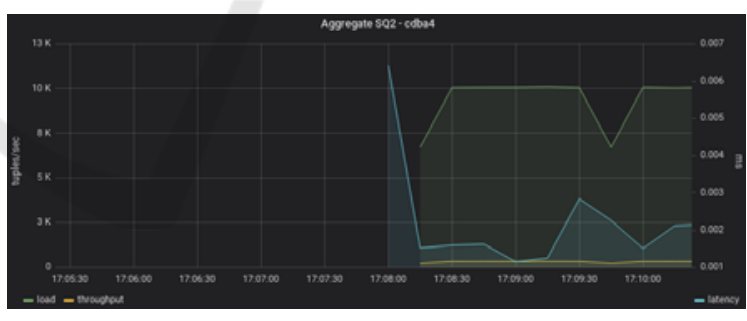

Figure 15: Query migration.

For the evaluation of the fault-tolerance protocol we present the behaviour of the data source and the data sink. The data source is in charge of duplicating the tuples and sending them to both nodes. The data sink receives the output tuples from the two nodes and outputs a single one. The first time a tuple arrives from one of the nodes, it outputs that tuple and keeps it in memory till the duplicate arrives or a failure happens. In the former case the tuple is eliminated, while in the latter case, the tuples are directly send to the client. 


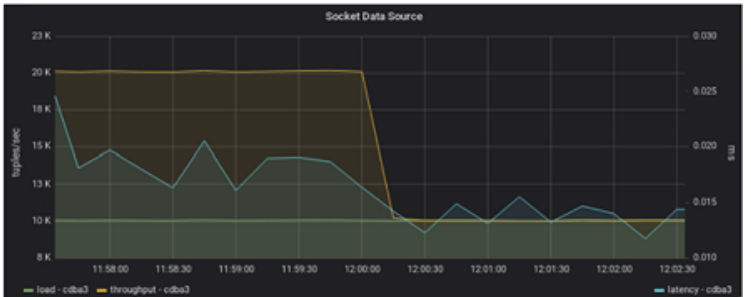

Figure 16: Data Source.

Figure 16 shows that the data source receives 10 000 tuples and sends 20000 tuples while there are two replicas, then a failure happens and it only sends the tuples to the available replica.

Figure 17 shows the number of tuples the data sink receives while the two replicas are running (680 tuples), when the failure happens one of the replicas stops sending tuples and therefore, the data sink receives 340 tuples per second. In both the former case it filters duplicates and sends half of the tuples. While in the latter case it sends the same amount of tuples but, there is no filtering.

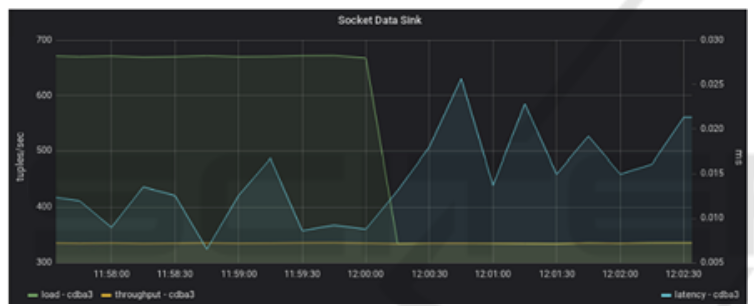

Figure 17: Data sink.

\section{CONCLUSIONS}

In this paper we have presented the migration and fault-tolerance protocols of UPM-CEP and their performance running the HiBench benchmark.

As future work we plan to implement other faulttolerance protocols providing more relaxed semantics.

\section{ACKNOWLEDGEMENTS}

This work has received funding from the European Union's Horizon 2020 research and innovation programme under grant agreements No 732051, 779747, the Madrid Regional Council, FSE and FEDER, projects Cloud4BigData and EDGEDATA (grants S2013TIC2894, S2018/TCS-4499), the œMinistry of Economy and Competitiveness
(MINECO) under project CloudDB (grant TIN201680350).

\section{REFERENCES}

Ahmad, Y. e. (2005). Distributed Operation in the Borealis Stream Processing Engine. ACM SIGMOD International Conference on Management of Data, 882-884.

Foundation, A. S. (2015). Apache Storm. Obtenido de http://storm.apache.org/

Fundation, T. A. (2010). Apache ZooKeeper. Obtenido de https://zookeeper.apache.org/

Fundation, T. A. (2014). Apache Flink ${ }^{\circledR}$ - Stateful Computations over Data Streams. Obtenido de https://flink.apache.org/

Gulisano, V. e. (2010). StreamCloud: A Large Scale Data Streaming System. 126-137.

Gulisano, V. e. (2012, 12 23). StreamCloud: An Elastic and Scalable Data Streaming System. IEEE Transactions on Parallel Distributed Systems, 2351-2365.

Intel. (2017). HiBench. Obtenido de https://github. com/Intel-bigdata/HiBench

$\mathrm{Pu}$, C. e. (2001). Infosphere Project: System Support for Information Flow Applications. (25-34, Ed.) $\operatorname{SIGMOD(30),~25-34.~}$ 